\title{
Das Spiel mit Wiederholung und Variation bei Bodo Wartke und Christian Hirdes - durch müssen oder es einfach laufen lassen
}

\section{Wiederholung als Merkmal sprachlicher Einheiten und Äußerungen}

Innerhalb der Sprachwissenschaft wird das Prinzip der Wiederholung als wesentliches Merkmal bestimmter sprachlicher Einheiten oder sogar von Sprache an sich herausgestellt. Dies unterstreicht der vielzitierte Satz aus Hermann Pauls Prinzipien der Sprachgeschichte, „Erst wo Sprechen und Verstehen auf Reproduktion beruht, ist Sprache da.“ (Paul [1880] 1920, Kap. 12, §131), der auf die Entstehung von Sprachen Bezug nimmt. Darüber hinaus spielt Wiederholung aber auch in der Struktur bereits bestehender Sprachen eine zentrale Rolle. Coseriu unterstreicht die Bedeutung ,'fertige[r]' Zeichenkombinationen, die als ganze tradiert werden“ (Coseriu [1980] 2007: 107). Hierbei unterscheidet er noch einmal zwischen den „geflügelten Worten“ - literarischen oder nichtliterarischen Texten, die einen so großen Bekanntheitsgrad innerhalb einer Sprachgemeinschaft erreicht haben, daß man eine teilweise wörtliche Kenntnis dieser Texte bei vielen Angehörigen der Sprachgemeinschaft voraussetzen kann“ (Coseriu [1980] 2007: 108) - und der wiederholten Rede, die „all das“ umfasst, „was innerhalb einer Sprachtradition als festgefügte Wendung weitergegeben wird“ (Coseriu [1980] 2007: 107; die Grenze zwischen beiden Unterkategorien kann sich jedoch verwischen). Wiederholung kann demnach als zentrales Prinzip gelten, das sich in den Strukturen von Sprachen und im sprachlichen und sprachbezogenen Wissen der Mitglieder einer Sprachgemeinschaft vielfach manifestiert - zunächst ohne dass damit eine komische Wirkung erzielt oder intendiert würde (vgl. hierzu insgesamt auch Thun 1978; Lang 1987).

Gleichzeitig können aber durch Wiederholungen sprachlicher Formen und Muster komische Effekte erzielt werden, was sich auch in der Häufigkeit von Wiederholungsstrukturen in unterschiedlichen Textsorten und Traditionen verbalen Humors widerspiegelt - man denke etwa an ironische Wiederholungen der Äußerungen des Gegenübers in Theaterstücken oder an Wiederholungsstrukturen im Witz (vgl. die Wiederholung des Musters der N+N-Komposita in Wolfshund und Ameisenbär mit der zugehörigen semantischen Interpretationsregel sowie die wiederholte Nennung von Eigennamen des Typs St. X und das zugehö- 
rige Schema, das etabliert wird: Stanna $\rightarrow$ St. Anna, Stoswald $\rightarrow$ St. Oswald, [Steiermark $\rightarrow$ ] St. Eiermark in den beiden nachfolgenden Beispielen).

Ein Igel trifft im Wald auf einen Wolfshund. Er fragt ihn:

„Oh, was bist Du denn für ein Tier?“

„Ich bin ein Wolfshund.“

„Ein Wolfshund?“

„Ja, mein Vater war ein Wolf und meine Mutter eine Hündin.“

„Ach so, sagt der Igel und geht weiter. Dann trifft er auf ein anderes Tier und fragt:

„Oh, was bist Du denn für einer?“

„Ich bin ein Ameisenbär.“

Der Igel überlegt eine Weile und sagt dann:

„Das glaub' ich dir nicht.“

(Koch, Krefeld und Oesterreicher 1997: 22)

Kanzler Kohl fährt nach Österreich in Urlaub. Am Zoll begrüßt ihn der Beamte und erkundigt sich, wohin der Kanzler fährt.

„Ich fahre nach Stanna“, sagt der Kanzler. Der Beamte stutzt und wünscht dann eine gute Reise. Im Auto erkundigt sich der Kanzler beim Chauffeur, was denn an seiner Antwort so komisch gewesen sei, woraufhin dieser ihm antwortet:

„Ach wissen Sie, Herr Bundeskanzler, in Österreich heißt St. immer Sankt.“

Im nächsten Jahr wiederholt sich dieselbe Geschichte, dieses mal mit St. Oswald, das der Kanzler als Stoswald ausspricht. Als der Beamte ihn wieder so eigenartig anschaut, fragt er abermals seinen Fahrer um Rat, der ihn ein zweites Mal aufklärt.

„Aha“, sagt der Kanzler, „dieses Mal werde ich’s mir merken.“

Als sie im dritten Jahr wieder am Zoll vorfahren, fragt der Zöllner wie immer höflich:

„Wo geht es dieses Jahr hin, Herr Bundeskanzler?“

Der Kanzler, ganz stolz darauf, daß er den Trick mit dem St. nicht vergessen hat, antwortet: „Dieses Jahr fahre ich nach Sankt Eiermark.“

(Koch, Krefeld und Oesterreicher 1997: 17-18)

Dabei zeigt sich die Bedeutung eines zweiten grundlegenden Prinzips, das mit dem der Wiederholung zusammenspielt: Es treten gewisse Veränderungen der Strukturen auf, durch die ein zuvor geprägtes Muster und eine dadurch erzeugte Erwartungshaltung des Rezipienten teilweise durchbrochen werden. So beruht der zweite Witz genau darauf, dass der Sprecher den Fehler in der dritten Szene vermeiden möchte und gleich die vermeintlich richtige Form verwendet.

Ebenso zeigt sich das Prinzip der Variation an sprachspielerischen Verfremdungen phraseologischer Einheiten - also wiederholter Rede im oben definierten sprachwissenschaftlichen Sinn -, die als Unterkategorie des Wortspiels gefasst werden (vgl. Winter-Froemel 2009: 1430-1431). Coseriu zitiert das Beispiel Viel Freund viel Schand (in Abwandlung von Viel Feind viel Ehr), das erst in Bezugnahme auf die implizite Ausgangswendung interpretiert werden könne im Sinne von „wer Auseinandersetzungen nur mit vielfältiger Unterstützung auszutragen 
in der Lage ist, erwirbt sich damit nicht eben hohes Ansehen“ (Coseriu [1980] 2007: 108). Häufig stellen phraseologische Einheiten auch den Ausgangspunkt für Texte dar, die diese Einheiten spielerisch wörtlich nehmen und variieren, wie etwa im Falle von Christian Morgensterns „Die weggeworfene Flinte“ (die Flinte ins Korn werfen) oder Christian Hirdes’ „Kopf verdreht“ („Du hast mir den Kopf verdreht, / wie es keine vor dir tat. / So um $180 \mathrm{Grad}$ / drehtest du das Ding herum. // Du hast mir den Kopf verdreht. / Das sieht ziemlich scheiße aus. / Einsam bleib’ ich nun zuhaus, / dreh' den Fernsehsessel um. [...]“).

Wiederholte Strukturen rufen somit vorherige Äußerungen auf, zu denen gleichzeitig eine Distanz erzeugt wird. Der Rezipient ist aufgefordert, die Äußerungen als Variation einer feststehenden Wendung, d. h. einer wiederholten Rede, zu begreifen. Gelingt dies, so entsteht ein Erfolgserlebnis; Sprecher bzw. Produzent und Hörer bzw. Rezipient stellen ihre sprachliche Kompetenz unter Beweis, was eine Art Verbrüderung bewirkt (vgl. den in der französischen Humorforschung etablierten Begriff der connivence, der betont, dass Produzent und Rezipient sich als auf einem gemeinsamen Niveau agierend erleben). Wiederholung im Zusammenspiel mit Variation kann insofern auch als Signal an den Rezipienten verstanden werden, das auf eine komische Dimension der Äußerung hinweist und eine entsprechende Interpretation der Äußerung nahelegt.

Ausgehend von diesen Überlegungen liegt meinem Beitrag die Annahme zugrunde, dass in Kommunikationskontexten verbalen Humors Wiederholungsstrukturen eine Fährte legen und die Rezipienten dazu einladen, sich auf ein Spiel mit Wiederholung und Variation einzulassen. Dies soll anhand von zwei Texten von Christian Hirdes und Bodo Wartke untersucht werden; ausgewählt wurden Texte, die jeweils von einem einzelnen idiomatischen Ausdruck - d. h. einem „klassischen“ Fall wiederholter Rede - ausgehen. Die Ausdrücke werden dabei bereits in den Titeln der Stücke genannt: Lass es einfach laufen und Da muß er durch. Analysiert werden soll, wie in den Texten durch Wiederholung der Ausdrücke in unterschiedlichen Kontexten neue Interpretationsmöglichkeiten ausgeschöpft werden, welche komischen Effekte durch die so entstehenden Variationen erzielt werden und welche weiteren sprachspielerischen Techniken begleitend zum Einsatz kommen.

\section{Christian Hirdes, Lass es einfach laufen}

Lass es einfach laufen (2008) von Christian Hirdes stellt einen Liedtext dar, der mit Klavierbegleitung präsentiert wird. Der Text besteht aus sechs Strophen, nach jeweils zwei Strophen folgt der Refrain. Wie der Titel des Stücks bereits 
andeutet, kommt dem genannten umgangssprachlichen Ausdruck (es/die Dinge/...) laufen lassen eine textkonstitutive Rolle zu; er stellt den Ausgangspunkt des Textes dar und gleichzeitig das Ziel, auf das die einzelnen Strophen zulaufen. Der Ausdruck kann dabei klar als phraseologisch eingestuft werden: Er weist eine polylexikalische Struktur auf, d.h. er ist aus mehreren Lexemen zusammengesetzt, er ist lexikalisiert, $d$. h. er besitzt eine konventionelle Bedeutung im Wortschatz des Deutschen, die im Duden als 'nicht eingreifen, sich nicht um etwas kümmern' (vgl. Duden s. v. laufen lassen, laufenlassen) angegeben wird. Der Ausdruck weist eine Festigkeit auf; er trägt als gesamter die entsprechende Bedeutung. Gleichzeitig besteht aber eine teilweise Austauschbarkeit der Elemente (vgl. die verschiedenen möglichen Realisierungen des Akkusativobjekts), und es können zusätzliche Elemente wie einfach eingeschoben werden (es einfach laufen lassen). Das Merkmal der Idiomatizität zeigt sich vor allem in der Umdeutung der Komponente laufen und der semantischen Ausbleichung des es, da der Phraseologismus auch verwendet werden kann, wenn im Kontext kein mögliches Substantiv im Neutrum vorhanden ist, auf welches das Pronomen bezogen werden könnte. Die Komponente lassen wird hingegen in der Ausgangsbedeutung verwendet, so dass der Ausdruck insgesamt als teil-idiomatisiert eingestuft werden kann.

Hieraus ergeben sich nun verschiedene Möglichkeiten spielerischer Umdeutungen, die in den einzelnen Strophen des Texts von Christian Hirdes realisiert werden. In den ersten beiden Strophen geht es um die idiomatische Bedeutung der Wendung, die dann auch durch den Text des Refrains zusätzlich unterstrichen wird, indem die Bedeutung durch die ebenfalls idiomatische Wendung es kommt, wie's kommt paraphrasiert wird und zusätzlich stützende Argumente genannt werden: „wie es kommt, kannst du nicht planen“; „das Schicksal wird sich seinen Weg alleine bahnen“:

Lass es einfach laufen

Wenn der gewohnte Trott dir manchmal auf die Nerven geht, und du schaust in den Spiegel und hoffst, dass da 'ne Antwort steht, lass es laufen. Lass es einfach laufen.

Wenn die im Fernseh'n sagen, die Welt ist schlecht, und die machen dir Angst.

Und du fragst dich dann, ob du wohl daran was ändern kannst,

lass es laufen. Lass es einfach laufen.

Lass es laufen. Lass es einfach laufen.

Es kommt, wie's kommt, und wie es kommt, kannst du nicht planen.

Lass es laufen. Lass es einfach laufen.

Das Schicksal wird sich seinen Weg alleine bahnen. 
Produktionsseitig wird damit zunächst die erwartete Bedeutung des Ausdrucks aufgerufen und für die Hörer etabliert. Bereits hier zeigen sich aber bereits auch semantische Effekte und kleine Unterschiede bei der Wiederholung der Wendung in den beiden Strophen: Die erste Strophe beschreibt die depressive Stimmungslage eines Individuums angesichts eines als eintönig empfundenen Alltags. Durch den situativen Kontext - den Blick in den Spiegel - wird gleichzeitig das Bild von Tränen evoziert, die die Wange hinunterlaufen, d. h. auch die Bedeutung 'fließen' der verbalen Komponente laufen klingt bereits an, und die Bedeutung der Wendung kann demnach hier paraphrasiert werden als 'lass die Tränen fließen, lass deinen Gefühlen freien Lauf' (vgl. lass es (einfach) raus), d. h. das Nichteingreifen (noch im Sinne der konventionellen Bedeutung der Wendung) bezieht sich auf einen konkreten physiologischen Vorgang (des Weinens), der nicht unterbrochen werden soll.

In der zweiten Strophe wird hingegen eine gesellschaftliche Dimension eröffnet: Der Text erwähnt das Massenmedium Fernsehen und die Aussage „die Welt ist schlecht“, die als zusammenfassende Kommentierung der Äußerungen z. B. eines Nachrichtensprechers oder einer Nachrichtensprecherin (im Text nur über die indefinite 3. Person Plural - „die im Fernseh'n“ - evoziert) interpretiert werden kann. Damit wird die konventionelle Bedeutung der Wendung nun in einem abstrakteren Sinn interpretiert; die Aussage „lass es (einfach) laufen“ empfiehlt den Verzicht auf gesellschaftliches Engagement, der durch die angedeutete zu erwartende Aussichtslosigkeit entsprechender Aktivitäten begründet wird. Die fatalistische Handlungsanweisung hat damit hier eine soziale und ethische Dimension und steht im Widerspruch zu allgemein anerkannten Handlungsprinzipien sozialer Gemeinschaften. So deutet sich an, dass die Aussage als ironisch (um-)interpretiert werden kann und eine „Mehrstimmigkeit“ der Äußerung vorliegt.

Der Begriff der Mehrstimmigkeit oder Polyphonie, der zunächst von Michail Bachtin in einem literaturwissenschaftlichen Kontext geprägt wurde, wird im Rahmen neuerer sprachwissenschaftlicher Ansätze für die Analyse von Äußerungen fruchtbar gemacht, bei denen mehrere „Stimmen“ zugleich zu sprechen scheinen: Es ist nicht nur eine Sprecherperson anzusetzen, sondern es liegen mehrere Äußerungsinstanzen vor, denen unterschiedliche - im Fall der Ironie sogar entgegengesetzte - Äußerungsabsichten zuzuschreiben sind (vgl. Ducrot 1984; Rabatel 2008). Im vorliegenden Fall zitiert bzw. inszeniert der Sprecher bzw. eine erste Sprecherinstanz - (von dem ich annehme, dass er die entsprechende Handlungsempfehlung nicht mitträgt) den fatalistischen Standpunkt einer anderen Äußerungsinstanz, zu dem eine Distanz besteht. Die Distanz bzw. Unstimmigkeit wird in der Darbietung des Liedes unterstrichen durch den sehr 
eingängigen und beschwingten Dur-Refrain, der im Kontrast zur zitierten negativen Kernaussage („die Welt ist schlecht“) steht. Damit wird auf sprachlicher und musikalischer Ebene eine Verunsicherung des Hörers herbeigeführt: Er muss die Mehr- bzw. Unstimmigkeit erkennen und die plausibelste eigentliche Aussageabsicht durch Umkehrung der Interpretation gemäß dem Prinzip der Ironie rekonstruieren. Festzuhalten bleibt, dass dennoch in den ersten beiden Strophen und dem folgenden Refrain die idiomatische Bedeutung der Wendung klar etabliert und gestärkt wird.

In der dritten Strophe wird sodann jedoch eine neue Verunsicherung des Hörers herbeigeführt bzw. ein zusätzlicher Verarbeitungsaufwand erzeugt, indem ein wörtliches Verständnis erzwungen wird: Hier referiert es nun auf das Substantiv Nachbarskind, von dem ausgesagt wird, dass es versucht, sich einem Tadel oder einer Bestrafung zu entziehen; auch das Verb laufen ist demnach in der wörtlichen Bedeutung (vgl. das Quasisynonym wegrennen) zu interpretieren:

Wenn dein Nachbarskind zum wiederholten Mal an deiner Türe schellt, und dann wegrennt, weil's das immer noch für unterhaltsam hält, lass es laufen. Lass es einfach laufen.

Der Handlungskontext ist hierbei ein relativ harmloser: Es handelt sich um einen Streich, der üblicherweise abgesehen von unnötiger körperlicher Bewegung des Haus- oder Wohnungsbesitzers zur Tür oder Sprechanlage keine weiteren negativen Folgen hat. Die Anweisung „lass es laufen“ empfiehlt eine großzügige Gelassenheit gegenüber dem Kinderstreich trotz dessen wiederholten Auftretens. Hier ist damit keine Polyphonie/Ironie mehr anzunehmen, die Interpretation wendet sich wieder zum unmarkierten (nicht polyphonen) Fall. Neu aber ist, dass dabei die wörtliche Interpretation der einzelnen Bestandteile der Wendung zugrunde gelegt werden muss, so dass gleichzeitig eine semantische Remotivierung der Wendung stattfindet (vgl. hierzu auch meinen Beitrag „Deutungsspielräume - Ambiguität und Motivation sprachlicher Zeichen als Quellen des Wortspiels“ im vorliegenden Band).

Die Interpretationsprinzipien kehren sich dann erneut um, wenn in der vierten Strophe eine weitere, zumindest teilkonventionalisierte Interpretation von es laufen lassen vorbereitet wird:

Wenn dich in der Bahn mit dem defekten Klo deine Blase quält, und im Bahnhof dann für'n Toilettengang dir das Kleingeld fehlt, lass es laufen. Lass es einfach laufen.

Lass es laufen. Lass es einfach laufen.

Es kommt, wie's kommt, und wie es kommt, kannst du nicht planen. 
Lass es laufen. Lass es einfach laufen.

Das Schicksal wird sich seinen Weg alleine bahnen.

Gemeint ist hier nun das Nichtzurückhalten des Urins, das auf einer anderen lexikalisierten Bedeutung von laufen - 'fließen' / 'Wasser, Flüssigkeit austreten, ausfließen lassen' (vgl. Duden, s. v. laufen, 9.a. / 9.b) beruht. (Interessanterweise wurde diese Bedeutung bereits in der ersten Strophe in Bezug auf den weniger stark tabuisierten Konzeptbereich des WEINENS vorbereitet.) Ein zusätzlicher Verarbeitungsaufwand für die Hörer entsteht dadurch, dass die Flüssigkeit, um die es im konkreten Fall geht, im Kontext nicht direkt genannt, sondern nur indirekt über andere Elemente des Erfahrungs- und Handlungszusammenhangs - in kognitiv-semantischer Terminologie dem entsprechenden Frame (vgl. Minsky 1975; Fillmore 1985) - evoziert wird (vgl. die im Kontext erwähnten Konzepte KLO, BLASE, TOILETTENGANG). Die Vermeidung der direkten Nennung des URINS erklärt sich dabei über den tabuisierten Status von Körperausscheidungen im Allgemeinen. Damit liegt hier eine Handlungsanweisung vor, deren Befolgung in der sozialen Interaktion klar sanktioniert würde und die folglich nicht als ernst gemeinte Aufforderung zu verstehen ist' ${ }^{1}$; damit kommt wiederum eine Mehrstimmigkeit der Äußerung ins Spiel. Zusätzlich kann aber auch eine (durchaus ernste) angedeutete Kritik des Sprechers am schlechten Wartungszustand vieler Züge der Deutschen Bahn gesehen werden, die das vorgestellte stigmatisierte Verhalten zumindest teilweise entschuldigen oder sogar als Zeichen eines bewussten Protests interpretierbar machen könnte.

Die letzten beiden Strophen beinhalten wiederum einen erneuten Bruch mit allen Interpretationsmustern, die bislang etabliert wurden. Die fünfte Strophe knüpft zunächst an die zweite an: Auch hier wird ein situativer Kontext von Fernsehnachrichten als Rahmen erzeugt, wobei durch die Verwendung des politisch nicht korrekten Ausdrucks Negerkind bereits wieder eine polyphone Dimension der Äußerung nahegelegt wird. Diese wird durch die nachfolgende Charakterisierung des angesprochenen $d u$ verstärkt, die das Bild eines träge auf dem Sofa sitzenden, Bier trinkenden, ordinären (vgl. die nachfolgende Äußerung „so'n Scheiß-Programm“) Fernsehzuschauers evoziert, der mit der Fernbedienung zwischen verschiedenen Kanälen zappt, um sich berieseln zu lassen. Sodann wird aber ein Bruch erzeugt, indem gerade nicht die erwartete Wendung lass es laufen wiederholt wird (wobei es auf das „Scheiß-Programm“ bezogen,

1 Der stark tabuisierte Charakter der Handlungsanweisung zeigt sich im Übrigen klar an Publikumsreaktionen beim Vortrag der vierten Strophe, so etwa bei einer Darbietung von Christian Hirdes im Theater Trier im Herbst 2015 anlässlich meiner Antrittsvorlesung an der Universität Trier. 
d. h. die Wendung wiederum deidiomatisiert wäre), sondern eine Aufforderung mit entgegengesetzter Bedeutung geäußert wird: „Steh auf!“ Angesichts des evozierten Stereotyps des Fernsehzuschauers kann angenommen werden, dass diese Handlung für diesen eine nicht unerhebliche Anstrengung bedeutet, die in Kauf genommen wird, um nicht weiter mit den verstörenden Fernsehbildern konfrontiert zu sein. Im Rahmen der polyphonen Interpretation potenziert sich so die in der zweiten Strophe angedeutete Kritik an fehlendem gesellschaftlichen Engagement. Es wird vor Augen geführt, wie sogar aktiver Aufwand betrieben wird, um zu vermeiden, zu einer Auseinandersetzung mit bestimmten Realitäten und möglicherweise sogar zu einer aktiven Positionierung angeregt zu werden.

Wenn die im Fernseh'n so'n dürres Negerkind zeigen mit so'm dicken Bauch, und dein Bier ist leer und die Batterie deiner Fernbedienung auch,

Steh auf! (Kannst du doch nicht laufen lassen, so'n Scheiß-Programm, und dein Bier kommt auch nicht von alleine.)

Die letzte Strophe schließlich bricht nicht nur inhaltlich, sondern auch formal mit dem Muster der Strophen 1-4. Der Text bricht hier ab, und nur die Instrumentalbegleitung vollendet die Strophe; der Refrain setzt erst wieder ein mit der Zeile „Es kommt, wie’s kommt“. Damit wird eine Lücke erzeugt, die vom Hörer zu schließen ist, indem er nach dem bekannten Muster „lass es laufen“ gedanklich ergänzt. In dem durch die Strophe vorbereiteten Kontext impliziert die Wendung dabei allerdings eine maximale Tabuverletzung im Sinne der Inkaufnahme der schweren Verletzung oder des Todes eines Kindes: Bezug nehmend auf das im Kontext erscheinende Substantiv Nachbarskind, das auf Strophe 3 zurückverweist, muss auch hier die wörtliche Bedeutung von es und laufen angesetzt werden; anders als in Strophe 3, wo es um ein für das Kind harmloses bzw. sogar positives Weglaufen geht (dort entgeht es dadurch einem Tadel oder einer Strafe), hat die Ortsveränderung hier nun lebensbedrohliche Konsequenzen, da sie ein Überqueren der Hauptverkehrsstraße beinhaltet (bezeichnenderweise werden wiederum die gefährlichen Objekte selbst - AUTOS, LKwS usw. - nicht explizit genannt). Der Hörer bzw. Zuschauer, der den entsprechenden Text „lass es laufen“ gedanklich ergänzt, wird in gewisser Weise zum Mittäter, da er selbst diese unerhörte Aussage formuliert und sich hierbei ertappt. Gleichzeitig befolgt der Liedtext durch die Schlusspointe auch das übergeordnete, durch den Titel der CD und des entsprechenden Programms von Christian Hirdes vorgegebene Thema „Lieder wo am Ende jemand stirbt“.

Doch wenn dein Nachbarskind, das dir im Rücken seiner Eltern gegenübersteht, auf der anderen Seite der Hauptverkehrsstraße den Eismann erspäht... 
...Es kommt, wie's kommt, und wie es kommt, kannst du nicht planen.

Lass es laufen. Lass es einfach laufen.

Das Schicksal wird sich seinen Weg alleine bahnen.

Lass es einfach laufen.

\section{Bodo Wartke, Da muß er durch}

Auch bei $D a$ muß er durch (2003) von Bodo Wartke handelt es sich um einen Liedtext, der mit Instrumentalbegleitung (ebenfalls Klavier) präsentiert wird. Die Liedform mit Strophen und wiederholtem Refrain erweist sich wiederum als optimal geeignet für ein Spiel mit Wiederholung und Variation. Dem Lied ist hier ein Einleitungstext vorangestellt, der die Verwendung des genannten Ausdrucks explizit motiviert: ${ }^{2}$

Da muß er durch

Dieses Lied ist das Ergebnis einer Wette, die ich mal besser nicht gewettet hätte. Ich hab gewettet, ich schreibe Euch ein Lied über jedes Thema, gebt mir einfach einen Titel.

Da meinte einer: „Ach, da hab' ich was für Dich! Wie wär's mit dem Titel 'Da muß er durch’?“ Drauf ich geflissentlich: „Auf 'durch' reimt sich aber überhaupt nix außer 'Lurch'. “Da grinst er mich nur an und sagt: „Tja, da mußt Du durch.“

Dem Künstler wird die Aufgabe gestellt, ein Lied (in sich reimenden Versen) zum genannten Thema zu schreiben, wobei das Fehlen von möglichen Reimwörtern außer der Tierbezeichnung Lurch als zentrale Schwierigkeit benannt wird. Die Äußerung des Gegenübers „da mußt Du durch“ stellt dabei ein Bild im Bild literaturwissenschaftlich nach Gide eine mise en abyme (vgl. Wilpert 2001: 525) dar: Das „Durchmüssen“ soll auf der Ebene des dargestellten Inhalts des zu schreibenden Lieds thematisiert werden, erscheint aber auch auf der Ebene der Textproduktion selbst.

Die Schwierigkeit mit dem formal möglichen Reimwort Lurch ergibt sich dabei aus dem relativ spezifischen semantischen Inhalt (Wortfeld Amphibien) und aus der Tatsache, dass der Ausdruck Lurch keine weiteren Bedeutungen

2 Nachfolgend wurden die rahmenden und kommentierenden Textteile durch Kursivierung kenntlich gemacht. Einzelne Schreibfehler wurden korrigiert; aus Gründen der besseren Lesbarkeit wurde dies nicht im Einzelnen hervorgehoben. Ebenso wurden in einigen Fällen Leerzeilen im Originaltext getilgt. Bei den Liedstrophen wurden zusätzliche Absätze eingefügt, um die Versgrenzen deutlicher hervorzuheben. 
besitzt ${ }^{3}$; die Reimlösung Lurch/durch wird dabei als zu einfach, bzw. zu weit hergeholt verworfen:

Na super, auf was hab'ich mich da eingelassen: Ich meine okay, klar, ich könnte jetzt ein Lied schreiben über einen Lurch, der irgendwo durch muß. Aber das wäre zu einfach. Diese Genugtuung will ich ihm nicht verschaffen.

Wie wird das Problem dann aber gelöst und die sprachliche Virtuosität, um die es in der Wette ging, unter Beweis gestellt? Im Folgenden werden hierzu sogar fünf Lösungen vorgestellt, die als „Versuche“ tituliert werden. Hierbei kommen unterschiedliche Verfahren zum Einsatz, wobei die jeweiligen Mängel der Verfahren explizit thematisiert werden, so dass eine ironische Brechung entsteht.

Erstens handelt es sich um ungenaue Reime, d. h. ein Spiel mit lautlicher Ähnlichkeit (Paronymie) anstelle eines Spiels mit lautlichem Gleichklang (Homophonie). Das Problem wird in der Kommentierung angezeigt („Sie haben sicherlich gemerkt, das Wort 'Storch' reimt sich nicht so gut auf 'durch'."), und es wird eine sehr fragwürdige Entschuldigung angeboten (,[...] dafür kann ich nichts. Daran sind im Grunde meine Eltern schuld. Die hätten mir ja auch erzählen können, die kleinen Kinder brächte der Lurch.“). Durch das Spiel mit der stereotypen Äußerung „die Kinder bringt der Storch“ liegt ein zusätzliches Spiel mit wiederholter Rede vor. Gleichzeitig lässt sich auch das Prinzip der Variation feststellen: Durch die Ersetzung des Ausdrucks „Storch“ durch „Lurch“ wird die Unvereinbarkeit der stereotypen Vorstellungen („bringt Kinder“, „fliegt im Winter in den Süden“) aufgezeigt und die Argumentation ad absurdum geführt. Zusätzlich findet sich in der ersten Strophe der Schüttelreim Schnabel nur / Nabelschnur, der zusätzlich Komik erzeugt. Eine weitere Quelle der Komik ist die Vermeidung des tabuisierten Ausdrucks Scheide, der von den Hörern zu ergänzen ist („na, Sie wissen schon“).

\footnotetext{
Als ich noch Kind war, hab' ich stets gedacht, uns kleine Kinder, die hat der Storch gebracht. Doch irgendwann begann ich mich zu fragen: Wie soll der arme Storch das alles tragen?

Wie transportiert er beispielsweise mit dem Schnabel nur außer dem Kind noch die Plazenta und die Nabelschnur?
}

3 Zwar existiert im österreichischen Sprachgebrauch auch die Form Lurch in der Bedeutung 'zusammengeballter, mit Fasern durchsetzter Staub' (vgl. Duden); diese kann aber vermutlich nicht bei einem breiten Publikum als bekannt vorausgesetzt werden. 
Bis ich erfuhr, die kleinen Kinder, die bringt doch nicht der Storch: Die kommen aus ... na, Sie wissen schon. Da mußt' ich durch!

Wobei, ich mußte da nicht durch, ich war ein Kaiserschnitt. Sie haben sicherlich gemerkt, das Wort „Storch“ reimt sich nicht so gut auf „durch“. Das stimmt, aber dafür kann ich nichts. Daran sind im Grunde meine Eltern schuld. Die hätten mir ja auch erzählen können, die kleinen Kinder brächte der Lurch. Haben sie aber nicht. Dabei wäre es für mich als Kind völlig egal gewesen, welches Tier den Job macht, ich hätte alles geglaubt. „Mama, sag mal, wo kommen eigentlich die kleinen Kinder her?“ „Die, mein Junge, bringt der Lurch.“ „Echt? Und wann hat der Lurch mich gebracht?“ „Im Sommer. Denn im Winter fliegen alle Lurche in den Süden.“ Ich schweife ab. Zurück zum Lied, zweiter Versuch:

Wenig später mußt' ich dann zur Bundeswehr.

Und die Bundeswehr ist ein gesundes Heer, hab' ich mir sagen lassen, denn dort treibt man eine ganze Menge Sport.

Nun kriech' ich schon seit Tagen durch den Matsch, mir ist kalt, ich bin dreckig und klatschnaß und beginne mich zu fragen: Was tu' ich hier nur? Ich weiß nur eins gewiß: Ich muß da durch.

Nurich-durch. Ja, ok.

Im zweiten Versuch kommt zusätzlich zur Manipulation der Aussprache noch ein Spiel an und über Wortgrenzen hinweg hinzu (Matsch/klatsch-[naß], tu' ich/durch). Der „problematische“ Reim wird zwar kommentierend wiederholt, anders als beim ersten Versuch bleibt eine mögliche Kritik am Reim hier jedoch implizit, und das Problem wird schnell übergangen, indem zur dritten Strophe übergeleitet wird. Neben dem Reim-Spiel lässt sich in der zweiten Strophe auch eine inhaltsbezogene Komik feststellen: Der Text spielt mit stereotypen (negativen) Vorstellungen bezüglich der Bundeswehr (vgl. auch die dargestellte Szene des Durch-den-Matsch-Kriechens), die in Kontrast gesetzt werden zu den Beschreibungen „gesundes Heer“ und „,dort treibt man eine ganze Menge Sport“; die Distanzierung wird ferner auch dadurch angezeigt, dass die Charakterisierung anderen Sprechern zugeschrieben wird („hab’ ich mir sagen lassen“). Auch hier wird so eine polyphone Interpretation der Äußerung nahegelegt.

Für die dritte Strophe wird vorab das verwendete Verfahren angekündigt: der Rückgriff auf eine regionale (hier norddeutsche) Aussprache. Entsprechende Rückgriffe kommen in sprachspielerischen Texten häufig zum Einsatz; auch dieses Verfahren wird aber als problematisch entlarvt, in diesem Fall aufgrund der Nichtübereinstimmung zwischen der regionalen Verortung der Aussprache und dem Ort des Geschehens, die innerhalb des deutschen Sprachraums annä- 
hernd maximal weit auseinander liegen (vgl. die vom Künstler bei der Darbietung des Liedes gesetzte Pause und die Publikumsreaktionen nach „In der Schweiz“):

Ich verspreche, die nächste Strophe reimt sich dafür jetzt aber auch perfekt, wenn man sie im norddeutschen Dialekt ausspricht. Ich bitte dabei zu entschuldigen, daß die Strophe rein inhaltlich überhaupt nichts mit Norddeutschland zu tun hat - eher im Gegenteil:

In der Schweiz regierte mal ein Landvogt, mit dem die Tyrannei in das Land zog, weil er die Schweizer munter unterjochte, weswegen man ihn nicht besonders mochte.

Wann wird der Retter kommen diesem Lande?

Schon sehr bald, denn der Vogt und seine Bande

sind grade auf dem Weg nach Küßnacht, da liegt seine Burg, doch auch eine hohle Gasse. Da muß er durch!

Ein zusätzlicher komischer Effekt ergibt sich dadurch, dass in dieser Strophe auf ein geflügeltes Wort aus Schillers Wilhelm Tell (1804) angespielt wird, in der das zentrale Stichwort der Reimaufgabe enthalten ist (,Durch diese hohle Gasse muss er kommen, / Es führt kein andrer Weg nach Küssnacht“, Wilhelm Tell, IV,3). Gemeinsam mit der folgenden Strophe liefert der Text eine Zusammenfassung der Schlüsselszene aus Schillers Drama. Innerhalb des inszenierten Wettspiels kann dies als Zeichen sprachlicher Virtuosität und somit als Teilerfolg des Sprechers verbucht werden. Ohne kommentierende Rahmung und in Fortsetzung des thematischen Zusammenhangs folgt sogleich ein weiterer Reim, der ebenfalls auf einer dialektalen Aussprache beruht (ruhig / durch).

Der Wilhelm Tell liegt hier schon auf der Lauer, und der ist auf den Landvogt ziemlich sauer, denn er wurde von ihm dazu gezwungen zu schießen, und zwar auf seinen eig'nen Jungen!

Nun hingegen zielt er ganz bewußt mit seinem Pfeil auf des Landvogts Brust.

Hier gilt es, Schütze, deine Kunst zu zeigen! Jetzt nur ruhig, Tell, stell dir vor, es wär ein Apfel! Da muß er durch!

Auch in der fünften Strophe beruht der Reim auf einer manipulierten Aussprache, hier eines Wortes griechischer Herkunft (Chirurg). Die entsprechende Aussprache ist nicht nur als regional bedingt, sondern kann als unangemessene lautliche Anpassung eines entlehnten Wortes und damit als Zeichen mangelnder sprachlicher Kompetenz interpretiert werden (zur möglichen komischen Wir- 
kung ungewöhnlich starker Lehnwortintegration vgl. z. B. Schweickard 1998: 294 und Winter-Froemel 2011: 317-318). Zusätzlich gibt es hier ein weiteres vertikales Wortspiel (Brett / Brad [Pitt]) und eine intertextuelle Anspielung auf ein anderes Stück von Bodo Wartke (Meine neue Freundin), deren erfolgreiches Erkennen ggf. eine Selbstbestätigung bewirken und die „Fan-Identität“ der Zuhörer des Lieds bestärken kann (vgl. den kommentierenden Hinweis „wie ich ja schon erwähnte“).

Auf die nächste Strophe hat mich eine Bekannte gebracht. Der hab ich das vorgesungen bis da, und die meinte „Hey, ich hab' die Lösung für Dein Problem. Schreib doch eine Strophe über Deine Freundin.“ „Häh?“ „Na über ihren Vater.“ „Was?“

„Na, ist doch klar: der Schönheitschirurg, das reimt sich doch extrem gut auf ,durch', zumindest dann, wenn man es nicht allzu sauber ausspricht, und das fällt doch sicher nicht so ins Gewicht.

Oder?“ Gut, keine schlechte Idee. Ich versuch's mal.

Mein Schwiegervater ist in seinem Job

- wie ich ja schon erwähnte - ziemlich top.

Doch neulich brauchte er sein ganzes Talent,

da war nämlich ein Sumo-Ringer sein Patient.

Sumo-Ringer kennen Sie?

Die sind ziemlich korpulent. Und der sagte, er hätt-

-e bitte gerne auch so einen Bauch wie'n Brett

... Pitt. Auf eine meterdicke Fettschicht schaut der Schönheitschirurg

und sagt zu sich: „Au backe, da muß ich durch!“

Die bisher besprochenen Strophen zeigen klar, dass bei Bodo Wartkes Text anders als bei Christian Hirdes - der Fokus zunächst auf der formalen Seite der sprachlichen Zeichen liegt. Doch auch hier wird gleichzeitig mit unterschiedlichen Bedeutungsdimensionen bzw. -optionen des Ausdrucks durch müssen gespielt. Dies wird in der obigen Strophe besonders deutlich, in der (unter zusätzlichem Einsatz des Stilmittels der Übertreibung) das Bild einer „meterdicken Fettschicht“ und damit eine sehr konkrete Vorstellung einer schwer zu durchdringenden Masse evoziert wird. Dabei wird im Wesentlichen mit zwei Bedeutungen gespielt: einerseits der idiomatischen Bedeutung 'eine schwere Aufgabe annehmen / durchstehen', andererseits der kompositionalen Interpretation 'ein konkretes materielles Hindernis bzw. eine konkrete Enge durchqueren müssen' (vgl. in den vorangehenden Strophen die Scheide bei der Geburt, 
Matsch, eine enge Gasse). ${ }^{4}$ Bemerkenswert ist ferner, dass sich die beiden genannten Interpretationen jeweils nicht ausschließen: Neben der Überwindung der materiellen Hindernisse geht es in allen Fällen auch um die Überwindung einer abstrakten Schwierigkeit. Anders als bei Christian Hirdes wird hier keine Uminterpretation des Ausdrucks erzwungen, sondern mehrere Bedeutungen und Interpretationsoptionen werden jeweils überlagert.

In der Folge zeigt sich jedoch, dass die initiale Herausforderung damit noch immer nicht als zufriedenstellend gelöst angesehen wird (die Wiederholung des Ausdrucks Schönheitschirurg durch den Wettpartner zunächst in der „falschen“, dann in der ,richtigen“ Aussprache veranschaulicht hierbei wiederum das in der Einleitung skizzierte Zusammenspiel von Wiederholung und Variation):

Als ich diese Strophe fertig hatte, habe ich sofort meinen Wettpartner angerufen und meinte: „Hier, fertig, fertig, hör's Dir an. “Ich hab's ihm vorgesungen am Telefon und meint: „Na, wie sieht's aus.“ Er sagte dann: „Moment mal, das reimt sich alles gar nicht.“ „Komm, da kann man doch mal'n Auge zudrücken.“ „Nein, Wette ist Wette! Wenn, muß es sich reimen. Schönheitschirurch! Es heißt Schönheitschirurg. Wenn Du willst, daß es sich reimt, mußt Du darauf achten, daß es am Ende gleich geschrieben wird!“

Der besserwisserische Rat des Wettpartners wird sodann aber ad absurdum geführt, womit ein weiterer Beweis der sprachlichen Virtuosität erbracht wird: Unter Rückgriff auf einen Ausdruck fremdsprachlicher Herkunft wird die behauptete Eindeutigkeit der Ausspracheregeln (etwa „wenn Wörter gleich geschrieben werden, werden sie auch gleich ausgesprochen“) falsifiziert, in dem die aus dem Englischen übernommene Aussprache von church unzulässigerweise auf durch übertragen wird ([t]3:tf] - [d3:t f]). Zusätzlich wird (ähnlich wie in der ersten Strophe) auf ein sexuelles Tabu angespielt, hier den Zölibat der katholischen Kirche, dessen Verletzung vom Publikum wiederum nur indirekt aus dem Verwandtschaftsverhältnis („mein Papa“) zu erschließen ist (vgl. auch die Vermeidung der expliziten Benennung des Geschlechtsverkehrs durch Ellipse des Verbs und das „weißt' schon“).

„Ach so, danke für’s Erklären. Ja, ich probier's nochmal!“

Nach der Bundeswehr war die Zeit reif, daß ich einen Beruf ergreif', und was ich werden wollte, war mir sofort klar:

katholischer Priester, genau wie mein Papa.

4 Lediglich bei der zweiten Tell-Episode stellt der Apfel nicht ein negatives, zu überwindendes Hindernis, sondern ein zu erreichendes Ziel dar. 
Doch der sagte: ,überleg’ Dir das genau, mein Sohn,

denn Du darfst dann eigentlich nicht mehr mit 'ner Frau, weißt' schon.

Denn das sind die Regeln in the Catholic Church!

Wenn Du das wirklich willst, mußt du da durch.“

Schließlich, als weiterer Beweis der sprachlichen Virtuosität und Schlusspointe, wird dann doch die eigentlich zu Beginn des Textes verworfene Reimlösung realisiert. Zusätzlich wird hier im kommentierenden Rahmen sozusagen en passant ein weiteres mögliches Reimwort erwähnt (zerfurch'). Gleichzeitig findet eine kunstvolle Engführung durch die dichte Aufeinanderfolge von Wortspielen statt, wobei zusätzlich ein Binnenreim (Kröten töten) und zwei weitere horizontale Wortspiele (Kröten 'Geld'/Kröten 'Amphibien' und weil sie Leichen sind, bevor sie laichen können) zum Einsatz kommen. Während in den anderen Strophen die idiomatisierte Bedeutung von durch müssen fast immer mitschwingt, kommt hier nun dem zu durchquerenden Objekt eine lebensrettende Funktion zu, so dass eine Uminterpretation erfolgt:

Dieser Song dauert nun schon fünf Minuten. Und dabei habe ich noch immer keinen guten richtigen Reim gefunden auf „,durch“, so sehr ich mir auch meine Stirn zerfurch'.

Mir scheint nichts anderes übrig zu bleiben, als eine letzte Strophe zu schreiben mit dem einzigen verbleibenden Reim auf „durch“. Sie wissen ja, welcher das ist. Genau.

Da muß ich dann wohl durch...

Da wir Deutschen ja so gerne mit dem Auto fahr'n, bauen wir öfter mal 'ne neue Autobahn.

Das kostet uns 'ne ganze Menge Kröten, weil Autos ja bekanntlich Kröten töten,

die jetzt nicht mehr ihr Laichgebiet erreichen können, weil sie Leichen sind, bevor sie laichen können.

Doch es gibt ja Gott sei Dank für jeden noch so kleinen Lurch heute Tunnel unter der Straße. Da muß er durch!

\section{Zusammenfassung}

Der Analyse der Texte von Christian Hirdes und Bodo Wartke zeigt ein gemeinsames Grundprinzip: Es werden jeweils für einen idiomatisierten Ausdruck Interpretationsspielräume ausgelotet, die über die im Sprachgebrauch konventionalisierte und damit erwartbare primäre Interpretation hinausgehen. Die Textsorte des Lieds erlaubt es dabei, innerhalb der Strophen jeweils neue Kontexte vorzubereiten und dann die Wendung innerhalb des wiederkehrenden Refrains 
(Christian Hirdes) bzw. am Ende der einzelnen Strophen (Bodo Wartke) zu präsentieren. Die Wiederholung der Wendung konstituiert die Pointe der jeweiligen Textabschnitte, wobei die variierten Interpretationen, die vom Kontext erzwungen werden, einen zusätzlichen Verarbeitungsaufwand und einen Überraschungseffekt erzeugen können (vgl. Publikumsreaktionen sowie die von den Künstlern bei der Performanz eingesetzten Gestaltungsmittel wie Sprechtempo, Pausen, Betonung, Lautstärke, die weiterführend näher untersucht werden könnten). Gleichzeitig zeigen die Texte, dass trotz des gemeinsamen Grundprinzips die Umsetzung in den Texten variiert. Indem noch weitere Mittel zur Erzeugung sprachlicher Komik begleitend eingesetzt werden (u. a. weitere Wortspiele, das Spiel mit unterschiedlichen Tabus, dialektaler Aussprache sowie das Spiel mit Mehrstimmigkeit und Ironie), ergibt sich ein enges Zusammenwirken unterschiedlicher sprachspielerischer Verfahren, unter denen Wiederholung und Variation eine wichtige Rolle zukommt.

\section{Literaturangaben}

\section{Untersuchte Texte}

Christian Hirdes, Kopf verdreht. Vom Künstler zur Verfügung gestellte Textfassung. Christian Hirdes, Lass es laufen. Vom Künstler zur Verfügung gestellte Textfassung.

Audiofassung veröffentlicht auf: Christian Hirdes, Lieder wo am Ende jemand stirbt, 2008 Satyr Records.

Bodo Wartke, Da muß er durch (2003). Text nach: Achillesverse - live in Berlin. https://www.bodowartke.de/medien (Zugriff 20.07.2018). Audiofassung veröffentlicht auf: Bodo Wartke, Achillesverse - Live in Berlin. Klavierkabarett im Reimkultur, Reimkultur Musikverlag, 2013.

\section{Weitere zitierte Literatur}

Coseriu, Eugenio. [1980] 2007. Textlinguistik. Eine Einführung. Herausgegeben und bearbeitet von Jörn Albrecht. 4. Aufl. (Tübinger Beiträge zur Linguistik 500). Tübingen: Narr.

Ducrot, Oswald. 1984. Le dire et le dit. Paris: Éditions de Minuit.

Duden online. https://www.duden.de/woerterbuch (Zugriff am 06.09.2018).

Fillmore, Charles. 1985. Frames and the Semantics of Understanding. Quaderni di semantica 6. 222-254.

Koch, Peter, Thomas Krefeld \& Wulf Oesterreicher. 1997. Neues aus Sankt Eiermark. Das kleine Buch der Sprachwitze. München: Beck.

Lang, Jürgen. 1987. Wortbildung und wiederholte Rede (anhand spanischer und deutscher Beispiele). In Wolf Dietrich, Hans-Martin Gauger \& Horst Geckeler (Hgg.), Grammatik und 
Wortbildung romanischer Sprachen. Beiträge zum Deutschen Romanistentag in Siegen, 30.9. -3.10.1985 (Tübinger Beiträge zur Linguistik, 297), 171-186. Tübingen: Narr.

Minsky, Marvin. 1975. A Framework for Representing Knowledge. In Patrick H. Winston (Hg.), The Psychology of Computer Vision, 211-288. New York: McGraw-Hill.

Paul, Hermann. [1880] 1920. Prinzipien der Sprachgeschichte. 5. Aufl. Tübingen: Niemeyer. http://gutenberg.spiegel.de/buch/prinzipien-der-sprachgeschichte-2742/1 (Zugriff 06.09.2018).

Rabatel, Alain. 2008. Homo narrans: Pour une analyse énonciative et interactionnelle du récit. 2 Bde. Limoges: Éditions Lambert-Lucas.

Schiller, Friedrich. 1804. Wilhelm Tell. gutenberg-spiegel.de/buch/wilhelm-tell-3332/15 (Zugriff am 10.09.2018).

Schweickard, Wolfgang. 1998. Englisch und Romanisch. In Günter Holtus, Michael Metzeltin \& Christian Schmitt (Hgg.), Lexikon der romanistischen Linguistik. Bd. VII: Kontakt, Migration und Kunstsprachen: Kontrastivität, Klassifikation und Typologie, 219309.Tübingen: Niemeyer.

Thun, Harald. 1978. Probleme der Phraseologie. Untersuchungen zur wiederholten Rede mit Beispielen aus dem Französischen, Italienischen, Spanischen und Rumänischen (Beihefte zur Zeitschrift für romanische Philologie 168). Tübingen: Niemeyer.

Wilpert, Gero von. 2001. Sachwörterbuch der Literatur. 8. Aufl. Stuttgart: Kröner.

Winter-Froemel, Esme. 2009. Wortspiel. In Gert Ueding (Hg.), Historisches Wörterbuch der Rhetorik. Vol. 9, 1429-1443. Tübingen: Niemeyer.

Winter-Froemel, Esme. 2011. Entlehnung in der Kommunikation und im Sprachwandel. Theorie und Analysen zum Französischen (Beihefte zur Zeitschrift für romanische Philologie 360). Berlin \& Boston: De Gruyter.

Esme Winter-Froemel ist Professorin für Romanistische Sprachwissenschaft an der Universität Trier. Ihre Schwerpunkte in Forschung und Lehre umfassen Sprachwandel, Sprachkontaktphänomene, lexikalische Semantik, Semiotik und Ambiguität sowie das Wortspiel. Seit 2013 leitet sie das wissenschaftliche Netzwerk „Dynamik des Wortspiels: Sprachkontakt, sprachliche Innovation, Sprecher-Hörer-Interaktion“ (DFG), in dessen Rahmen bei De Gruyter die Buchreihe „The Dynamics of Wordplay“ erscheint. 
